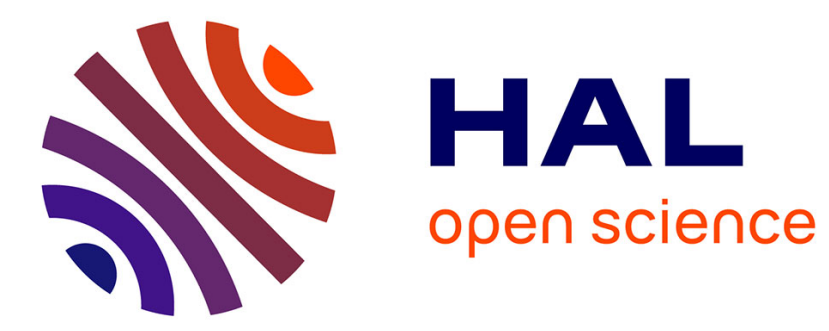

\title{
MPQ-trees for the orthogonal packing problem
}

Cédric Joncour, Arnaud Pêcher, Petru Valicov

\section{To cite this version:}

Cédric Joncour, Arnaud Pêcher, Petru Valicov. MPQ-trees for the orthogonal packing problem. Journal of Mathematical Modelling and Algorithms, 2012, 11 (1), pp.3-22. 10.1007/s10852-0119159-z . hal-00611528

\section{HAL Id: hal-00611528 \\ https://hal.science/hal-00611528}

Submitted on 30 Jul 2011

HAL is a multi-disciplinary open access archive for the deposit and dissemination of scientific research documents, whether they are published or not. The documents may come from teaching and research institutions in France or abroad, or from public or private research centers.
L'archive ouverte pluridisciplinaire HAL, est destinée au dépôt et à la diffusion de documents scientifiques de niveau recherche, publiés ou non, émanant des établissements d'enseignement et de recherche français ou étrangers, des laboratoires publics ou privés. 


\title{
MPQ-trees for the orthogonal packing problem
}

\author{
Cédric Joncour, Arnaud Pêcher, Petru Valicov
}

July 26, 2011

\begin{abstract}
Given a set of rectangular items of different sizes and a rectangular container, the aim of the bi-dimensional Orthogonal Packing Problem (OPP-2 for short) is to decide whether there exists a non-overlapping packing of the items in this container. The rotation of items is not allowed. In this paper we present a new exact algorithm for solving OPP-2, based upon the characterization of solutions using interval graphs proposed by Fekete and Schepers. The algorithm uses MPQ-trees, which were introduced by Korte and Möhring to recognize interval graphs.
\end{abstract}

Keywords Orthogonal Packing Problem · MPQ-trees

Let $V$ be a set of $D$-dimensional rectangular shapes. For $d \in\{1, \ldots, D\}$ and every $v \in V$, let $w_{d}(v) \in \mathbb{Q}^{+}$ (resp. $w_{d}(C)$ ) be the length of $v$ (resp. of the container $C$ ) with respect to the dimension $d$. For every subset of items $S \subseteq V$, let $w_{d}(S)=\sum_{v \in S} w_{d}(v)$. Let $W(v)=\prod_{d=1}^{D} w_{d}(v)$ and $W(C)=\prod_{d=1}^{D} w_{d}(C)$ be the volumes of the item $v$ and of the container $C$ respectively.

The $D$-dimensional orthogonal packing problem (OPP- $D$ ) is to decide if the set of items $V$ fits into the container $C$ without overlapping (if true, $V$ is said to be feasible). Formally speaking, we have to find out whether $\forall d \in\{1, \ldots, D\}$ there exists a function $x_{d}: V \rightarrow \mathbb{Q}^{+}$, such that:

$$
\begin{gathered}
\forall v \in V, x_{d}(v)+w_{d}(v) \leq w_{d}(C) \\
\forall v_{1}, v_{2} \in V,\left(v_{1} \neq v_{2}\right),\left[x_{d}\left(v_{1}\right), x_{d}\left(v_{1}\right)+w_{d}\left(v_{1}\right)\right) \cap\left[x_{d}\left(v_{2}\right), x_{d}\left(v_{2}\right)+w_{d}\left(v_{2}\right)\right)=\emptyset
\end{gathered}
$$

Let $p_{v} \in \mathbb{Q}^{+}$be the value associated with an item $v \in V$. The $d$-dimensional knapsack problem (OKPd) consists in computing a feasible set $V^{\prime} \subseteq V$ such that $\sum_{v \in V^{\prime}} p_{v}$ is maximal.

In this paper, we consider the bi-dimensional case, which has been the most studied so far [11, 9, 6, $12,7,3,5,8,1]$. This paper is organized as follows. In the first section we describe Fekete and Schepers' model and point out some issues which motivated our work. In the second section we define the model of MPQ-trees (introduced in [17]) and give an algorithm to check feasibility. In the third section, we present the computational results compared with other algorithms on standard benchmarks. The fourth section is a short conclusion.

This research was partially supported by the ANR Project GraTel ANR-09-blan-0373-01.

C. Joncour

IMB / INRIA Bordeaux - Sud-Ouest, University of Bordeaux, 351 Cours de la Libération, 33405 Talence Cedex, France

E-mail: cedric.joncour@math.u-bordeaux1.fr

A. Pêcher

IRIT, University of Toulouse, 118 Route de Narbonne, 31062 Toulouse Cedex 9, France

E-mail: pecher@irit.fr

P. Valicov

LaBRI, 351 Cours de la Libération, 33405 Talence Cedex, France

E-mail: valicov@labri.fr 


\section{Fekete and Schepers' model}

In this paper, all graphs are simple, undirected and finite. We denote by $N(u)$ the set of neighbours of the vertex $u$. $C_{n}$ denotes a cycle on $n$ vertices. A 2-chordless cycle is a cycle $v_{0}, \ldots, v_{n-1}, v_{0}$ such that there is no edge $v_{i} v_{j}$ for $i, j \in\{0, \ldots, n-1\}$ and $|i-j| \bmod n=2$. A stable or independent set of a graph is a subset of pairwise non-adjacent vertices of this graph. A clique is a set of pairwise adjacent vertices. An interval graph is a graph $G=(V, E)$ such that there is an assignment of intervals $I_{v}(v \in V)$ of the real line to the vertices of $G$ such that for every pair of vertices $u$ and $v, u v$ is an edge if and only if $I_{u} \cap I_{v} \neq \emptyset$.

Given a feasible packing of a set of items $V$, for every dimension $d$, let $G_{d}=\left(V, E_{d}\right)$ be the interval graph with vertex set $V$ and edge set $E_{d}$, such that $i j$ is an edge if and only if the projections of the packing of items $i \in V$ and $j \in V$ onto the dimension $d$ intersect (see Fig. 1 for an illustration).
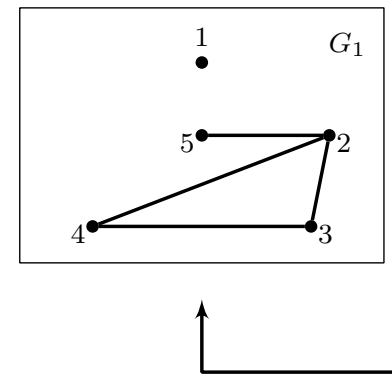
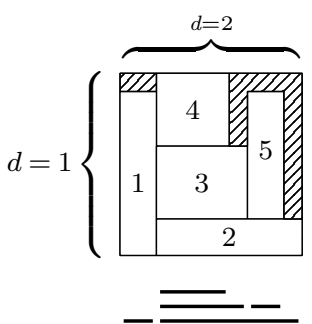
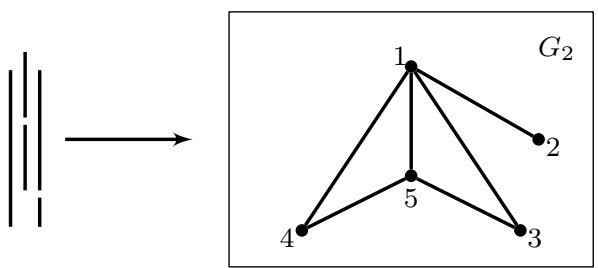

Fig. 1: Example of 2D packing and its associated interval graphs

Fekete and Schepers proved the following result, which is crucial for our algorithm:

Theorem 1 (Fekete and Schepers [10]) Given a D-dimensional container $C$, a set of items $V$ is feasible, if and only if there is a set of $D$ graphs $G_{d}=\left(V, E_{d}\right)$, with $d \in\{1, \ldots, D\}$, such that:

(P1) Every graph $G_{d}$ is an interval graph

(P2) For every stable set $S$ of $G_{d}, w_{d}(S) \leq w_{d}(C)$

(P3) $\bigcap_{d=1}^{D} E_{d}=\emptyset$

The tuple of the $D$ graphs $G_{d}$ is called a packing class.

Fekete, Schepers and van der Veen gave an efficient algorithm for solving OKP- $D$ by solving its subproblem OPP-D [12]. Their algorithm is based upon the packing classes. To do so, they used the following characterization of interval graphs:

Theorem 2 (Ghouilà-Houri [15], Gilmore and Hoffman [16]) A graph $G$ is an interval graph if and only if it does not contain an induced $C_{4}$ and its complement does not contain a 2-chordless cycle of odd length.
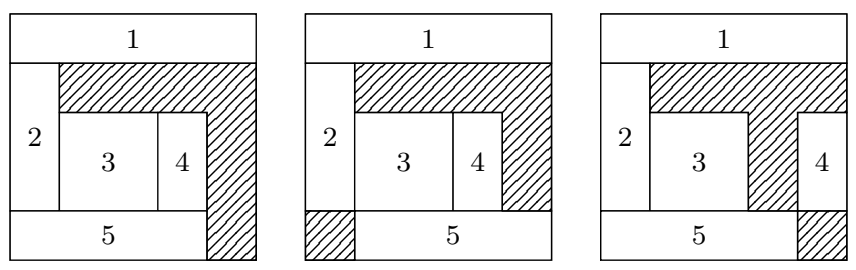

Fig. 2: Symmetrical solutions in Fekete and Schepers' model 
Despite its efficiency, their algorithm may enumerate symmetrical solutions. An example is given in Figure 2 where "almost" similar packing configurations are modeled by different couples of interval graphs. Moreover, there are some degeneracy issues of Fekete and Schepers' algorithm pointed out in [13], implying the generation of some unnecessary couples of interval graphs.

\section{Our approach}

Our aim is to handle those symmetry issues more efficiently, by taking advantage of the nice algorithmic properties of the MPQ-tree data structure. Fekete and Schepers' model considers only the intersection of the projections of the items onto each dimension. In our approach we maintain some extra information that enables the early detection of some symmetrical solutions or some infeasible packing configurations. This was the motivation to use a different representation of interval graphs and we chose MPQ-trees for this purpose. In this section we recall the definition of an MPQ-tree by presenting the idea of the algorithm of recognition of interval graphs using this data structure introduced in [17] and give a new characterization of feasible items. We give an algorithm to solve OPP-2 using MPQ-trees and provide some optimizations by exploiting some properties of feasible sets of items.

\subsection{MPQ-trees}

To mention the relationship between MPQ-trees and interval graphs we need the following definition:

Definition 3 Let $G=(V, E)$ be a graph and $Q_{1}, \ldots, Q_{m}$ its maximal cliques. A consecutive arrangement of the maximal cliques of $G$ is an order $\prec$ over the maximal cliques such that $Q_{i} \prec Q_{j}$ if:

$\forall v \in V$, if $v \in Q_{i}$ and $v \in Q_{j}$, then $v \in Q_{k}$ for all $k$ s.t. $Q_{i} \prec Q_{k} \prec Q_{j}$.

Theorem 4 (Fulkerson and Gross [14]) A graph $G$ is an interval graph if and only if the maximal cliques of $G$ can be linearly ordered to obtain a consecutive arrangement.

Let $M$ be a set of elements and $\mathcal{M}$ a collection of subsets of $M$. A PQ-tree is a data-structure representing all permutations of $M$ that are consistent with constraints of consecutiveness given by $\mathcal{M}$ with the convention that the elements of each $M^{\prime} \in \mathcal{M}$ must occur consecutively in a permutation. In the case of interval graphs, $M$ is the set of maximal cliques, and $\mathcal{M}$ is the set of all $\mathscr{C}(v), v \in V$, where $\mathscr{C}(v)$ denotes the set of all maximal cliques containing $v$.

A PQ-tree is a planar drawing of a rooted tree with two types of internal vertices: $\mathrm{P}$ and $\mathrm{Q}$, represented by circles and rectangles respectively. The leaves of a PQ-tree are labelled 1-1 with the maximal cliques of an interval graph $G$. To avoid ambiguity, we will call nodes, the vertices of a PQ-tree.

The frontier $F(T)$ of a PQ-tree $T$, represents the permutation of the maximal cliques obtained by the ordering of the leaves of $T$ from left to right. A PQ-tree $T^{\prime}$ is equivalent to $T$, if one can be obtained from the other by applying the following rules a finite number of times:

1. Arbitrarily permute the children of a P-node

2. Reverse the order of children of a Q-node
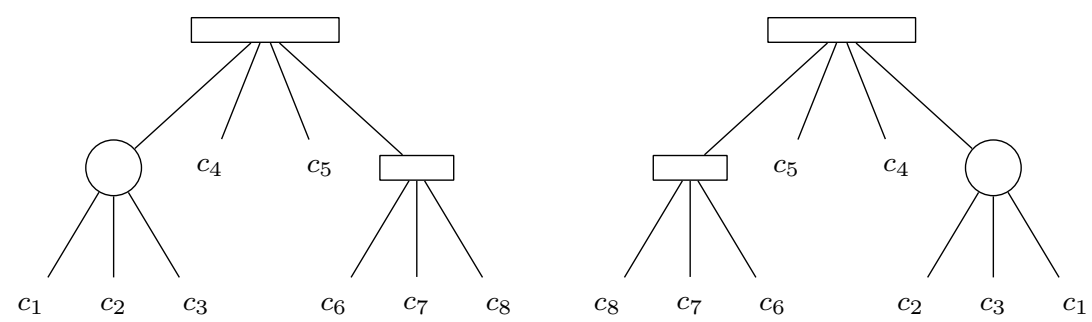

Fig. 3: Equivalent PQ-trees 
Figure 3 shows an example of two equivalent PQ-trees.

A proper $\mathrm{PQ}$-tree is one for which the $\mathrm{P}$-nodes have at least two children and the Q-nodes have at least three children. From now on, by the term PQ-tree we will consider a proper PQ-tree.

Theorem 5 (Booth and Lueker [4]) A graph $G$ is an interval graph if and only if there exists a $P Q$-trees $T$ such that $F(T)$ is a consecutive arrangement of the maximal cliques of $G$.

A modified $P Q$-tree (MPQ-tree, introduced by Korte and Möhring in [17])) associated with a graph $G$ is an extension of a PQ-tree where the nodes of the tree are labelled with some subsets of vertices of $G$, such that each branch of the MPQ-tree represents a maximal clique. A P-node is assigned only one set, while a Q-node is assigned a set for each of its children. Here are the rules of the labelling:

- A P-node is labelled by the set of vertices of $G$ which are only contained in all cliques represented by the subtree of $T$ rooted in this node.

- A leaf is labelled by the set of vertices of $G$ contained only in the clique represented by this leaf.

- A Q-node, with $m$ children $F_{1}, \ldots, F_{m}$, is labelled by a list of sets $S_{k}$, for $k \in\{1, \ldots, m\}$, each of them being called a section such that a section $S_{k}$ corresponds to the child $F_{k}$ in the left-to-right order. Each section $S_{k}(k \in\{1, \ldots, m\})$ contains the vertices of $G$ contained in all cliques represented by the subtree rooted in $F_{k}$ and also in all cliques represented by the subtree rooted in another child $F_{l}$ $(l \in\{1, \ldots, m\}$ and $l \neq k)$.

We will say that a node $N$ of an MPQ-tree associated with a graph $G$ contains a vertex $v$ of $G$ if $v \in V_{N}$, where $V_{N}$ is the vertex set or section (in case of a Q-node) of the label of $N$. Notice that from the definition of an MPQ-tree, a vertex $v$ of $G$ is contained in exactly one MPQ-tree node. However, $v$ can be contained in more than one section of a Q-node.
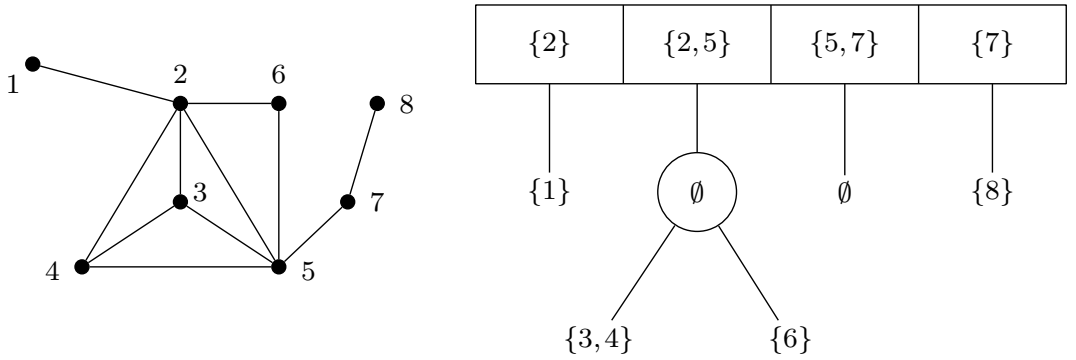

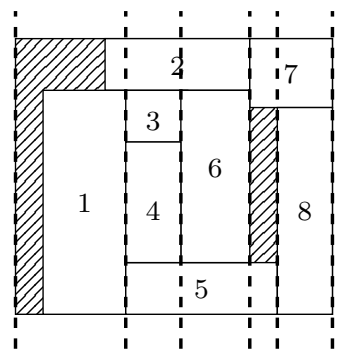

Maximal cliques: $\{1,2\},\{2,3,4,5\},\{2,5,6\},\{5,7\},\{7,8\}$

Fig. 4: An interval graph, an associated MPQ-tree and the packing configuration

Figure 4 shows an example of an MPQ-tree associated with an interval graph $G$, where both representations modelize the intersections in dimension 1 of a feasible packing in terms of maximal cliques (the intersections are given by the strips in the picture).

We have the following characterization of interval graphs, which is the basis of our algorithm:

Theorem 6 (Korte and Möhring [17]) A graph $G$ is an interval graph if and only if there exists an $M P Q$-tree associated with $G$.

Hence we may consider MPQ-trees instead of interval graphs since Theorem 6 gives an equivalence between the two structures.

Lemma 7 (Korte and Möhring [17]) Let $G$ be an interval graph and $T$ its associated MPQ-tree. Then $G+u$ (where $u$ is a vertex added to $G$ ) is an interval graph if and only if the following holds:

1. All vertices adjacent to $u$ are contained in a unique path of $T$.

2. For each $Q$-node $N$, labelled with sections $S_{1}, \ldots, S_{m}$, let $S=S_{1} \cup \ldots \cup S_{m}$. Then $S \cap N(u) \in S_{1}$ or $S \cap N(u) \in S_{m}$ 
In other words, Lemma 7 says that while building an MPQ-tree incrementally from an interval graph (i.e. adding vertices of the graph one by one) only one path of this tree must be updated and due to the properties of the reading orders of children of nodes of an MPQ-tree, this path can be chosen to be the leftmost one. This restricts considerably the number of cases to consider while updating the MPQ-tree. This result is crucial for our algorithm.

Using Theorem 6, Korte and Möhring gave an algorithm recognizing if a graph $G$ is an interval graph, by constructing an associated MPQ-tree. It uses a LexBFS-ordering (introduced in [18]) $\lambda=\left[v_{1}, \ldots, v_{n}\right]$ of the vertices of $G$ to iteratively build the MPQ-tree.

Let $G=(V, E)$ be an interval graph with an associated MPQ-tree $T_{G}$ and $u$ the vertex to be inserted in $T_{G}$ to obtain the MPQ-tree $T_{G+u}$ associated with the graph $G+u$. Since vertices are inserted in LexBFS-order, $N(u)$ must induce a clique. Let $N$ be a node of $T_{G}$. If $N$ is of type $\mathrm{P}$ or a leaf, let $V_{N}$ be its associated vertex set and if $N$ is a Q-node let $V_{N}$ be the vertex set corresponding to the first section. The principle of Korte and Möhring's algorithm is the following:

- Find the unique path $P$ of the current MPQ-tree having a node $N$ such that $V_{N} \cap N(u) \neq \emptyset$. This path can be either leftmost or rightmost.

- Find the first node $N_{*}$ in the bottom-up traversal such that $\exists j \in V_{N_{*}}, j \in N(u)$.

- Select the corresponding pattern and apply the suitable replacement: let $V_{N_{*}}=A \cup B$ be the partition of $V_{N_{*}}$ such that $A=V_{N_{*}} \cap N(u)$ and $B=V_{N_{*}} \backslash A$. Let $N^{*}$ be the highest node in $P$ such that $V_{N^{*}} \backslash N(u) \neq \emptyset$ if it exists and let $N^{*}=N_{*}$ otherwise. Due to Lemma 7 , the patterns which can be applied are described in Figures 5, 6 and 7. In the case when $N_{*} \neq N^{*}$ the patterns are applied recursively by rewriting the current tree starting from $N_{*}$ up to $N^{*}$, to obtain a valid MPQ-tree. We omit details which are rather technical. For an elaborate explanation, see the original paper [17]. Notice that the process is deterministic e.g. at each step only one pattern can apply. If no pattern can be applied to the current configuration, then $G+u$ is not an interval graph.

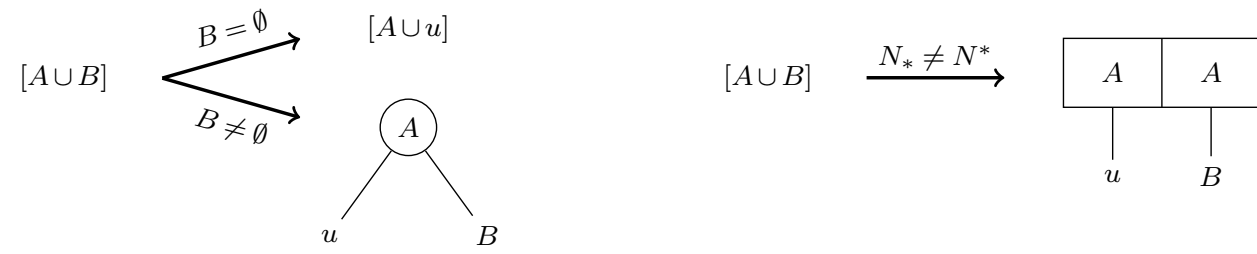

Fig. 5: Templates of a leaf $\left(V_{N_{*}}=A \cup B\right)$

Definition 8 For every node $N$ of an MPQ-tree associated with a dimension $d$ of a packing configuration, we define the width $\lambda_{N}$ of $N$ as described below.

- If $N$ is a leaf $L$ labelled with the set $V_{L}, \lambda_{L}=\max _{i \in V_{L}}\left\{w_{d}(i)\right\}$ if $V_{L} \neq \emptyset$ and $\lambda_{L}=0$ otherwise.

- If $N$ is a P-node $P$ labelled with the set $V_{P}$, and $\lambda_{f_{1}}, \ldots, \lambda_{f_{m}}$ are the widths of each of its children, $\lambda_{P}=\max \left\{\sum_{j=1}^{m} \lambda_{f_{j}}, \max _{i \in V_{P}}\left\{w_{d}(i)\right\}\right\}$

- If $N$ is a $\mathbf{Q}$-node $Q$, let $S_{1}, \ldots, S_{m}$ be its sections and $\lambda_{f_{1}}, \ldots, \lambda_{f_{m}}$ the widths of each of the children of the node. In order to define the width $\lambda_{Q}$, we first define, recursively from $m$ to 1 , the widths of its sections: $\lambda_{S_{j}}, \forall 1 \leq j \leq m$ :

$$
\lambda_{S_{m}}=\lambda_{f_{m}}
$$

Suppose $\lambda_{S_{k+1}}, \ldots, \lambda_{S_{m}}$ are defined, then

$$
\lambda_{S_{k}}=\max \left\{\lambda_{f_{k}}, \max _{i \in S_{k}, i \notin S_{k-1}}\left\{w_{d}(i)-\sum_{h>k, i \in S_{h}} \lambda_{S_{h}}\right\}\right\}
$$



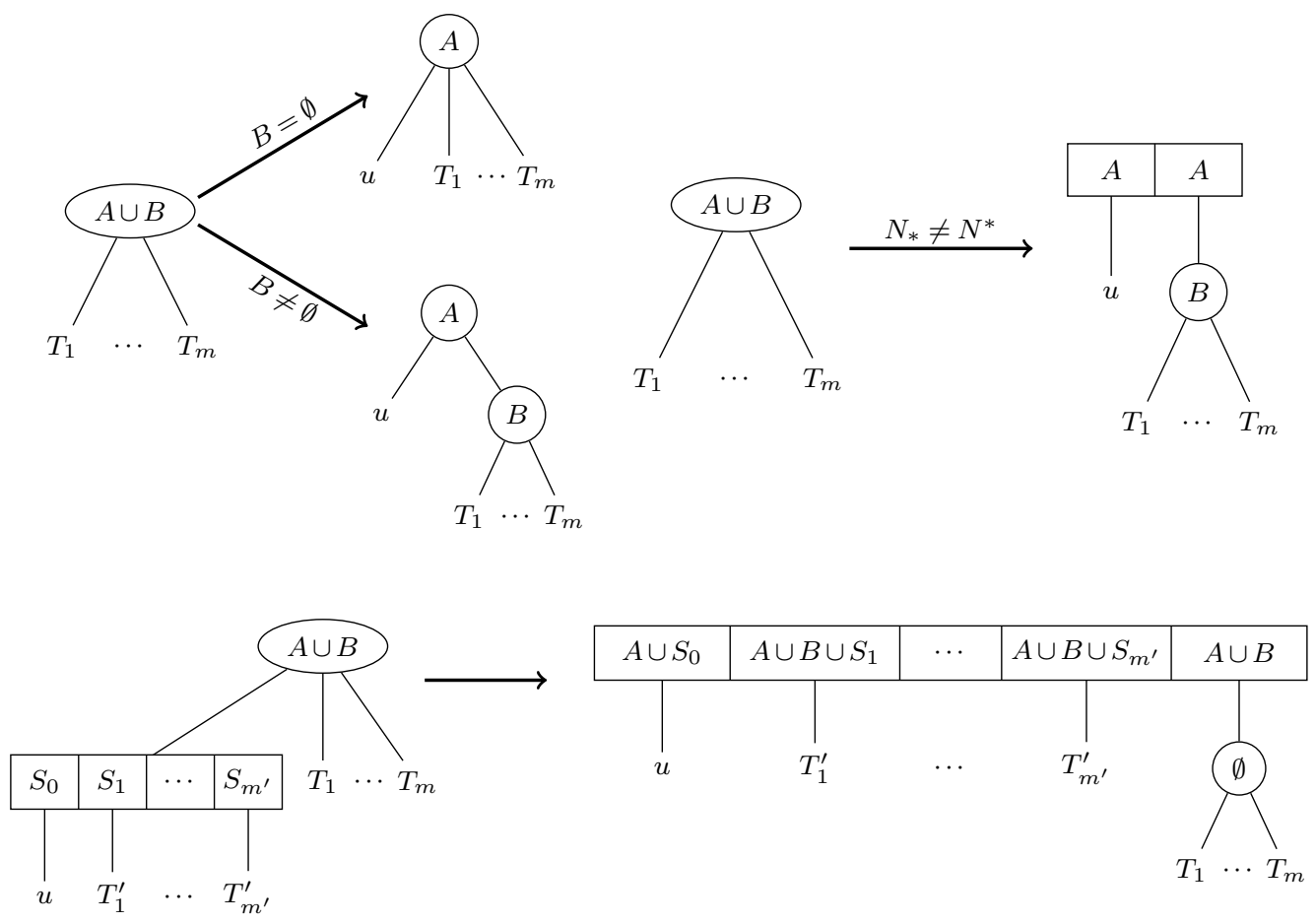

Fig. 6: Templates of a P-node

The width of $N$ is given by the following formula

$$
\lambda_{Q}=\sum_{k=1}^{m} \lambda_{S_{k}}
$$

It follows from definition that for every node $N$ with children $f_{1}, \ldots, f_{m}, \lambda_{N} \geq \sum_{k=1}^{m} \lambda_{f_{k}}$.

Lemma 9 Let $G_{d}=\left(V, E_{d}\right)$ be an interval graph of a packing class and $T_{d}$ an associated $M P Q$-tree. Let $v$ be an item of $V$. For every $P$-node or leaf $N$ such that $v$ belongs to the label of $N$, we have $w_{d}(v) \leq \lambda_{N}$. For every Q-node $N$ containing $v$ in the labels of some of its sections, we have $w_{d}(v) \leq \sum_{h=k}^{l} \lambda_{S_{h}}$, where $S_{k}, \ldots, S_{l}$ are the sections of $N$ containing $v$.

Proof The cases of a P-node and of a leaf are obvious. Suppose $N$ is a Q-node with sections $S_{1}, \ldots, S_{m}$ and children $f_{1}, \ldots, f_{m}$. Since branches associated with sections $S_{k}, \ldots, S_{l}$ of $N$ are all the cliques containing $v$, we have $\lambda_{S_{k}} \geq w_{d}(v)-\sum_{h=k+1}^{l} \lambda_{S_{h}}$. Hence, $w_{d}(v) \leq \sum_{h=k}^{l} \lambda_{S_{h}}$.

The following proposition translates property (P2) of Theorem 1.

Proposition 10 Let $G$ be an interval graph. $G$ satisfies property $P 2$ if and only if for every associated $M P Q$-tree $T$ of $G$, the width $\lambda_{R}$ of the root of $T$ verifies $\lambda_{R} \leq w_{d}(C)$.

Proof

Suppose $G$ satisfies property P2 and consider an MPQ-tree $T$ of $G$ with root $R$. We will prove that $\lambda_{R} \leq w_{d}(C)$.

The proof is by induction on the distance between $R$ and the nodes of $T$. Let $H(k)$ be the assertion: "If $N$ is a node of $T$ at distance $k$ from $R$ then there is a stable set $S$ of $G[N]$ such that $\lambda_{N} \leq w_{d}(S)$, where $G[N]$ is the subgraph of $G$ induced by the set of vertices of $G$ contained in the labels of the subtree of $\mathrm{T}$ rooted in $N . "$ 

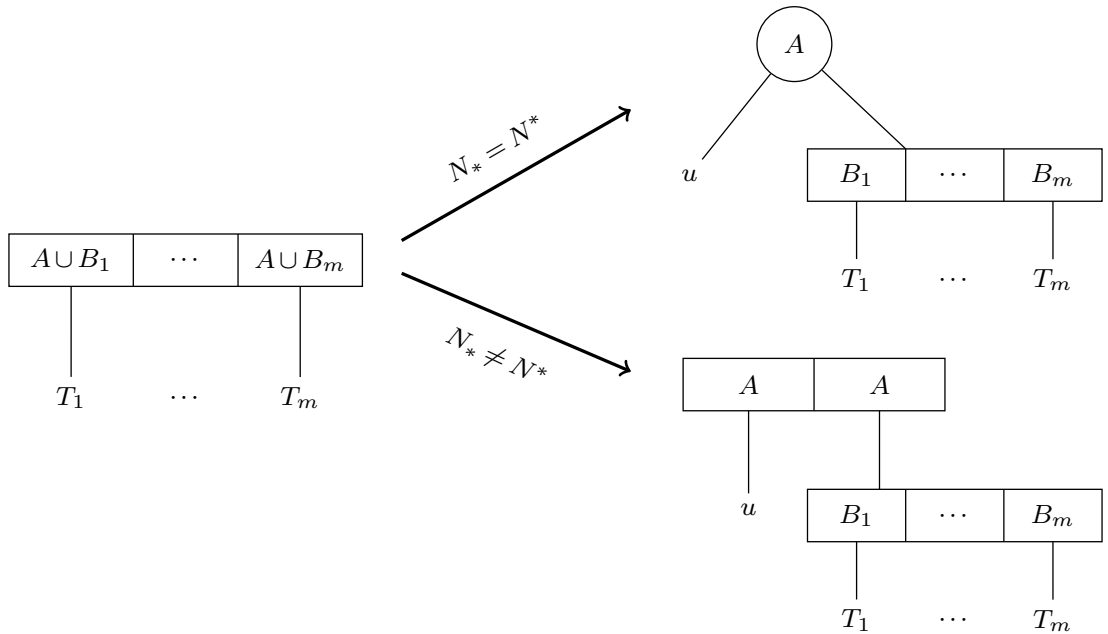

(a) Q1
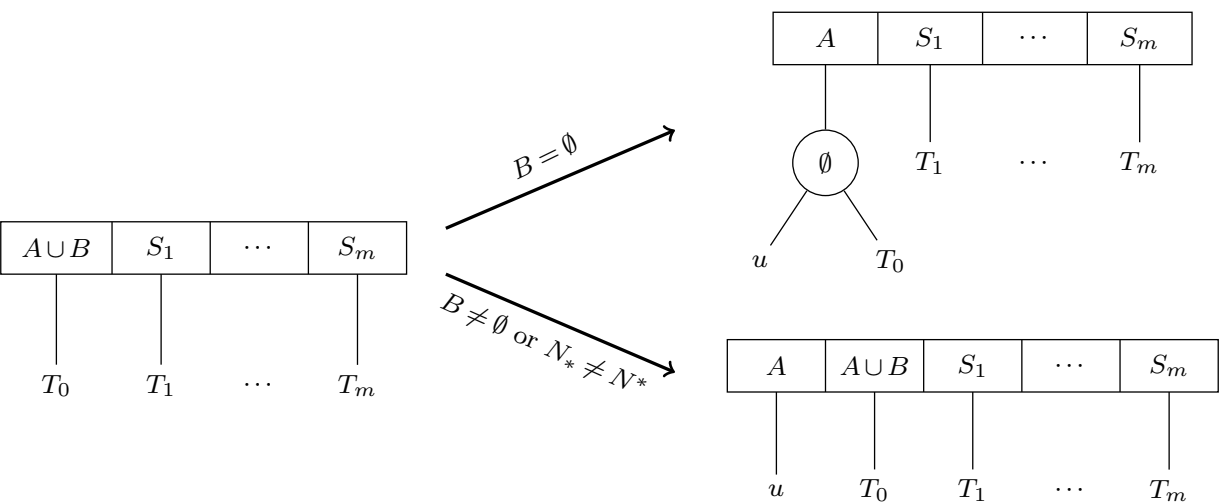

(b) Q2
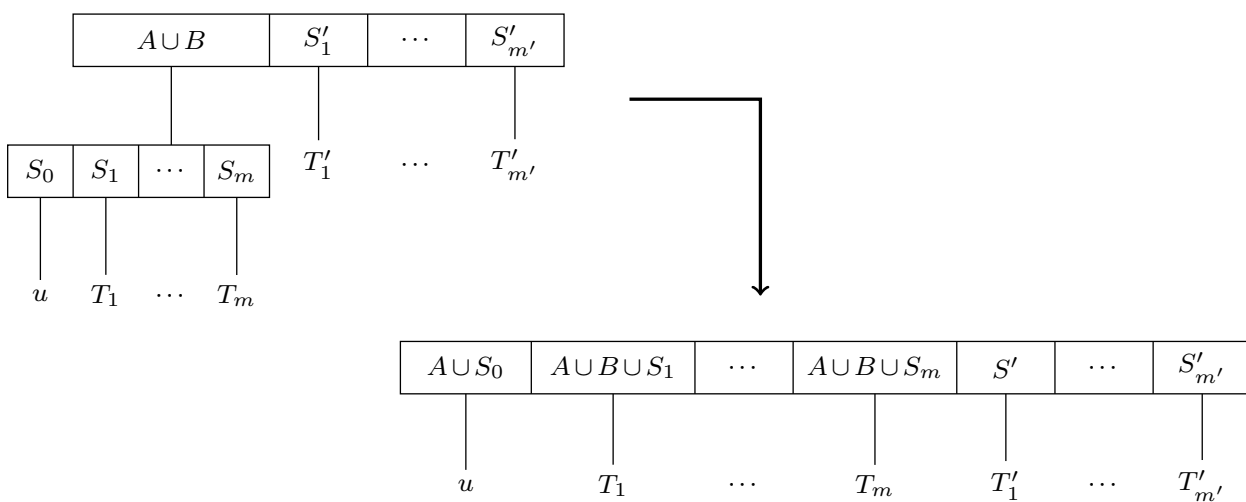

(c) Q3

Fig. 7: Templates of a Q-node

- Let $M$ be the maximal distance from $R$ in $T$. Let $N$ be any node of $T$ at distance $M$ from $R$. Hence $N$ is a leaf. Due to the definition of a leaf, there is $i$ in $V_{N}$ such that $w_{d}(i)=\lambda_{N}$. Hence, $S=\{i\}$ and $H(M)$ is true.

- Assume that $H(k)$ is true for $k \leq M$ (induction hypothesis). Let $N$ be any node of $T$ at distance $k-1$ from $R$.

- If $N$ is a leaf then the above argument applies again. 
- If $N$ is a P-node with $m$ sons, then we distinguish two cases. If $\max _{j \in V_{N}}\left\{w_{d}(j)\right\} \leq \sum_{k=1}^{m} \lambda_{f_{k}}$, then let $S=\bigcup_{k=1}^{m} Z_{k}$, where $Z_{k}$ is the stable set associated to son $f_{k}$ such that, due to induction hypothesis, $\lambda_{f_{k}} \leq w_{d}\left(Z_{k}\right)$. Since in the graph $G_{d}$ there are no edges between two vertices contained in two different children of $N, S$ is clearly an independent set of $G[N]$. Applying the induction hypothesis, $\lambda_{N} \leq \sum_{k=1}^{m} \lambda_{f_{k}} \leq w_{d}(S)$ and we are done. If $\max _{j \in V_{N}}\left\{w_{d}(j)\right\}>\sum_{k=1}^{m} \lambda_{f_{k}}$ then let $S=\{i\}$ where $i$ is such that $w_{d}(i)=\max _{j \in V_{N}}\left\{w_{d}(j)\right\}$. Thus $\lambda_{N}=w_{d}(S)$ and we are done.

- If $N$ is a Q-node with sections $S_{1}, \ldots, S_{m}$ then let $Z_{k}$ be the stable set of $G$ built by iteration of $k$ from 1 to $m$ :

- $k=1$. If the width of $S_{1}$ is equal to the width of its child $f_{1}$, then due to the induction hypothesis, $Z_{1}$ is a stable set of $G[N]$ such that $\lambda_{S_{1}} \leq w_{d}\left(Z_{1}\right)$; otherwise there is a vertex $b \in S_{1}$ such that $\lambda_{S_{1}}=w_{d}(b)-\sum_{h>1, b \in S_{h}} \lambda_{S_{h}}$, in which case define $Z_{1}=\{b\}$.

- $k \geq 2$. If there is a vertex $b \in S_{k}, b \in S_{k-1}$ and $Z_{k-1}=\{b\}$, define $Z_{k}=\{b\}$; otherwise, if there is a vertex $b \in S_{k}, b \notin S_{k-1}$ and $\lambda_{S_{k}}=w_{d}(b)-\sum_{h>k, b \in S_{h}} \lambda_{S_{h}}$, then $Z_{k}=\{b\}$; otherwise, necessarily $\lambda_{S_{k}}=\lambda_{f_{k}}$, in which case, due to the induction hypothesis, $Z_{k}$ can be chosen to be the stable set of $G\left[f_{k}\right]$ such that $\lambda_{f_{k}} \leq w_{d}\left(Z_{k}\right)$.

Let $S=\bigcup_{k=1}^{m} Z_{k}$. Clearly, $S$ is a stable set of $G_{N}$ such that $\lambda_{N}=\sum_{k=1}^{m} \lambda_{S_{k}} \leq \sum_{s \in S} w_{d}(s)=w_{d}(S)$.

Hence $H(k-1)$ is true.

Due to the induction, $H(0)$ is true, and since $w_{d}(S) \leq w_{d}(C)$ (property P2 of Theorem 1), we have $\lambda_{R} \leq w_{d}(C)$.

Conversely, suppose $\lambda_{R} \leq w_{d}(C)$. Consider an independent set $S$ of $G_{d}$. By definition of an MPQ-tree, $\forall u \in S$, in $T_{d}$ there exist exactly two distinct cases for the node $N$ of $T_{d}$ containing $u$ :

1. $N$ is a Q-node. Let $S_{k}, \ldots, S_{l}$ be the sections of $N$ containing $u$. In this case, let $\Lambda_{u}=\sum_{h=k}^{l} \lambda_{S_{h}}$.

2. $N$ is a P-node or a leaf. In this case, let $\Lambda_{u}=\lambda_{N_{u}}$.

Since $S$ is an independent set, no two vertices of $S$ are contained in the same branch of $T_{d}$. Hence, by definition of $\lambda_{R}$, we have $\sum_{s \in S} \Lambda_{s} \leq \lambda_{R_{d}}$. By Lemma 9, we have $\forall x \in V, w_{d}(x) \leq \Lambda_{x}$. Hence, $w_{d}(S) \leq \lambda_{R_{d}}$. Applying the hypothesis, $w_{d}(S) \leq w_{d}(C)$ which is exactly the property (P2) of Theorem 1 .

Notice that property (P3) of Theorem 1 may be easily translated with respect to MPQ-trees, since the branches of an MPQ-tree represent the maximal cliques of the interval graphs.

\subsection{The core of the algorithm to check feasibility}

For a given dimension and a given order on the items (which are the vertices of the associated interval graph), the (pseudo) Algorithm 1 constructs all the MPQ-trees to get the one representing this dimension. Thus, it may have to be executed for all possible orders $\sigma$ (in the next subsection, we explain that we have in fact to consider only a subset of orders). The items are added one by one in the MPQ-tree such that for every node $N, \lambda_{N} \leq w_{d}(C)$.

\subsection{Optimizations}

The structure of an MPQ-tree provides some partial information about the position of the items which can be easily exploited. For instance, its frontier gives the order of appearance of items in the packing 


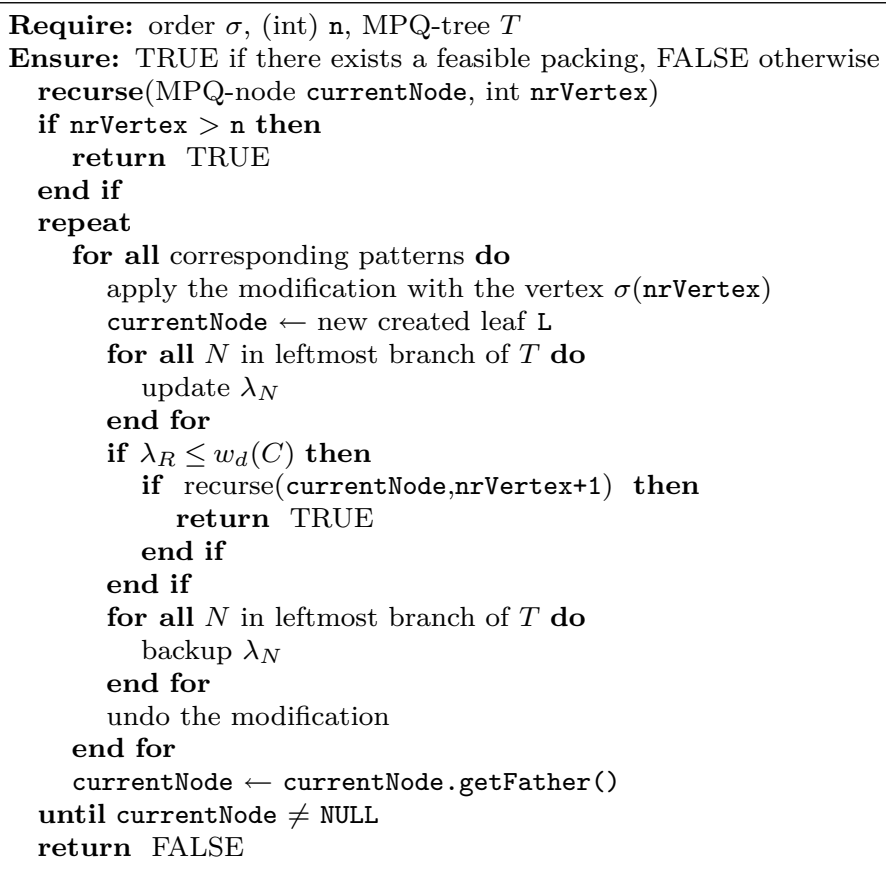

Algorithm 1: recursive enumeration of MPQ-trees to check feasibility

configuration. In addition, the higher an item is contained in the tree associated with a dimension $d$, the more items it "covers" in $d$ (i.e. the more are the intersections of its projection on $d$ with the projections of the other items on $d$ ). Being able to compute at each step the widths of each node, we provide some optimizations of the algorithm by adding some valid constraints, breaking some symmetries and early detecting some infeasible configurations.

\subsubsection{Bi-dimensional case: remaining areas, widths and branches heights}

In the bi-dimensional case, a few basic valid constraints may be applied when enumerating MPQ-trees in dimension 1 :

1. Assume that at the step $i$ of the algorithm, an MPQ-tree $T_{1}$ containing $\sigma(1), \ldots, \sigma(i)$ was constructed with the root having $m$ children $f_{1}, \ldots, f_{m}$. After the step $i$, the widths of the children $f_{2}, \ldots, f_{m}$ cannot be modified (since only the leftmost branch can be modified), therefore by Proposition 10, the remaining items $\sigma(i+1), \ldots, \sigma(n)$ have to be packed in the area $W(C)-w_{2}(C) * \sum_{h=2}^{m} \lambda_{f_{h}}$. Hence the following constraint is valid:

$$
\sum_{h=i+1}^{n} W(\sigma(h))+w_{2}(C) * \sum_{h=2}^{m} \lambda_{f_{h}} \leq W(C)
$$

2. The width of any item among $\sigma(i+1), \ldots, \sigma(n)$ cannot exceed the remaining width of the container after removing $\lambda_{f_{2}}, \ldots, \lambda_{f_{m}}$ :

$$
\sum_{h=2}^{m} \lambda_{f_{h}}+\max \left\{w_{1}(\sigma(i+1)), \ldots, w_{1}(\sigma(n))\right\} \leq w_{1}(C)
$$

3. Let $\mathcal{B}$ be a branch of $T_{1}$. Since all the items contained in this branch overlap in the dimension 2 , the sum of the size of these items in dimension 2 cannot exceed $w_{2}(C)$ :

$$
\sum_{i \in \mathcal{B}} w_{2}(i) \leq w_{2}(C)
$$




\subsubsection{Positive widths}

The left part of the Figure 8 is an example of packing which could be avoided by considering only the right part. Next lemma establishes a sufficient condition for avoiding the enumeration of this type of equivalent packing configurations. Hence, our algorithm will consider only the right packing of Figure 8.
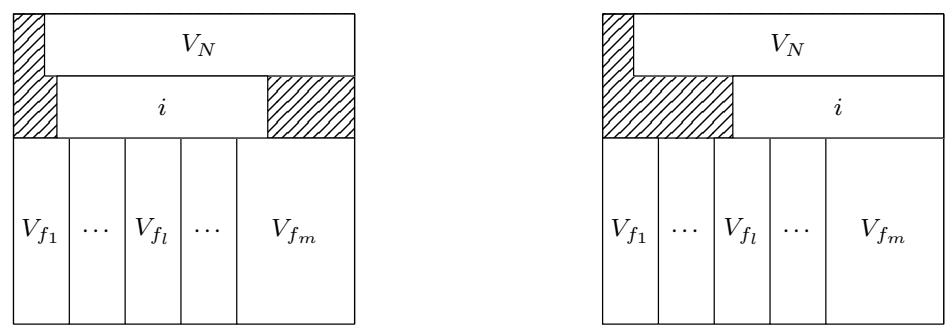

Fig. 8: Example of "similar" packing configurations

Lemma 11 Let $G_{d}$ be an interval graph of a packing class. There is an associated MPQ-tree of $G_{d}$ such that for every internal node $N$ with $m$ sons $f_{1}, \ldots, f_{m}$, the following holds:

- If $N$ is a P-node, $\forall i \in V_{N}, w_{d}(i)-\sum_{k=2}^{m} \lambda_{f_{k}}>0$

- If $N$ is a Q-node with sections $S_{1}, \ldots, S_{m}, \forall k \in\{1, \ldots, m\}, \forall i \in S_{k}, w_{d}(i)-\sum_{h>k, i \in S_{h}} \lambda_{S_{h}}>0$

Proof Recall that, from Definition 8, for every node or section $X, \lambda_{X} \geq 0$.

Let $T^{\prime}$ be an MPQ-tree associated with $G_{d}$ and let $N^{\prime}$ be an internal node of $T^{\prime}$ not satisfying the statement.

- Suppose $N^{\prime}$ is a P-node. Then there is $i \in V_{N^{\prime}}$, such that $w_{d}(i)-\sum_{k=2}^{m} \lambda_{f_{k}} \leq 0$.

We choose $i$ such that $w_{d}(i)=\min _{j \in V_{N^{\prime}}}\left\{w_{d}(j)\right\}$. Let $l=\max \left\{h \geq 2 \mid w_{d}(i) \leq \sum_{k=h}^{m} \lambda_{f_{k}}\right\}$. We distinguish two cases for the value of $l$.
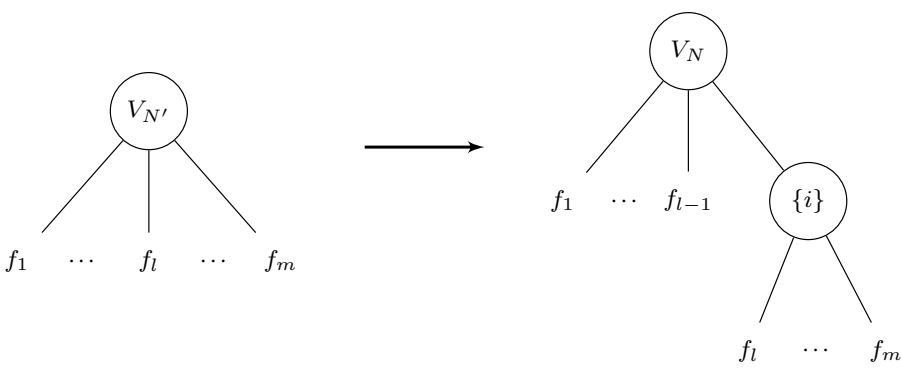

Fig. 9: P-node replacement

- Suppose $l<m$. Let $T$ be the MPQ-tree obtained from $T^{\prime}$ by replacing $N^{\prime}$ as shown in Figure 9 . Observe that the MPQ-trees associated to dimension 1 of the packing configurations of Figure 8 are the MPQ-trees of Figure 9 when $f_{1}, \ldots, f_{m}$ are leaves. In $T, N$ is the P-node replacing $N^{\prime}$ of $T^{\prime}$ and having $V_{N}=V_{N^{\prime}} \backslash\{i\}$ as a labelling set, $\{i\}$ is the labelling set of a new P-node, say $A$, 
having $f_{l}, \ldots, f_{m}$ as children. In $T, \lambda_{A}=\sum_{k=l}^{m} \lambda_{f_{k}}$ and, therefore, $\sum_{k=1}^{m} \lambda_{f_{k}}=\sum_{k=1}^{l-1} \lambda_{f_{k}}+\lambda_{A}$. Hence, $\lambda_{N}=\lambda_{N^{\prime}}$. Finally, we note that $w_{d}(i)-\sum_{k=l+1}^{m} \lambda_{f_{k}}>0$.

- Suppose $l=m$. Let $T$ be the MPQ-tree obtained from $T^{\prime}$ by removing $i$ from the set $V_{N^{\prime}}$ and modifing $f_{m}$ as follows. If $f_{m}$ is a leaf or a P-node, then insert $i$ in the labelling set of $f_{m}$. If $f_{m}$ is a Q-node insert $i$ in every section of $f_{m}$. Now, if $f_{m}$ is a leaf we are done. Otherwise, if $i$ does not satisfy the statement for $f_{m}$, then apply the proof on $f_{m}$.

- Suppose $N^{\prime}$ is a Q-node with sections $S_{1}^{\prime}, \ldots, S_{m}^{\prime}$. Then there are $i$ and a section $S_{k}^{\prime}$ with $i \in S_{k}^{\prime}$, such that $w_{d}(i)-\sum_{h>k, i \in S_{h}^{\prime}} \lambda_{S_{h}^{\prime}} \leq 0$. We choose $k$ such that $k=\min \left\{h \mid i \in S_{h}\right\}$. Let $l=\min \left\{h \mid w_{d}(i)>\right.$ $\left.\sum_{h^{\prime}>h, i \in S_{h^{\prime}}^{\prime}} \lambda_{S_{h^{\prime}}^{\prime}}\right\}$. We have $w_{d}(i)-\sum_{h \geq l, i \in S_{h}^{\prime}} \lambda_{S_{h}} \leq 0$. We distinguish two cases for the value of $l$.

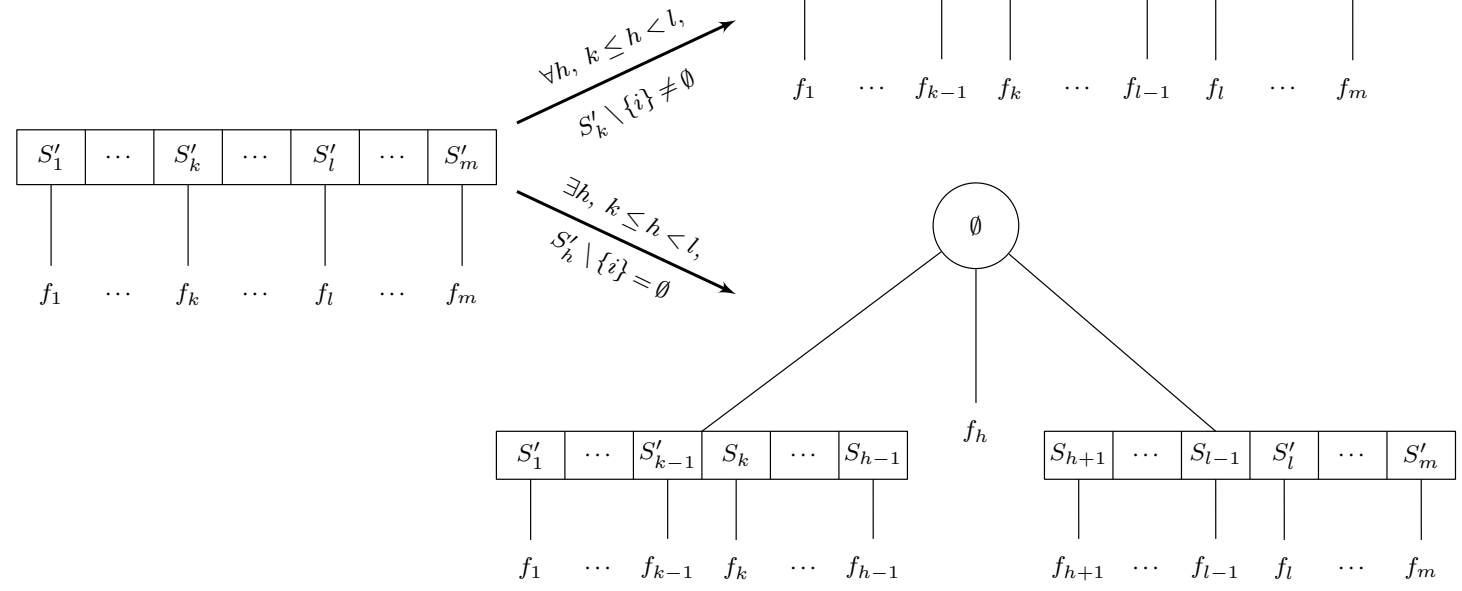

Fig. 10: Q-node replacement

- Suppose $l<m$. Intuitively, one has to remove $i$ from every section of $N^{\prime}$ which does not satisfy the statement of the lemma in case of a Q-node. Apply the replacement of Figure 10 to construct from $T^{\prime}$ an MPQ-tree $T$, in which $\forall h \in\{k, \ldots, l-1\}, S_{h}=S_{h}^{\prime} \backslash\{i\}$ and the other sections do not change. To distinguish the widths of $T$ and $T^{\prime}$, let $\lambda_{M}^{T}$ (resp. $\lambda_{M}^{T^{\prime}}$ ) denote the width of any node (or section) $M$ of $T$ (resp. of $T^{\prime}$ ).

Consider the upper case of the replacement of Figure 10. We have $\forall h \in\{l, \ldots, m\}, \lambda_{S_{h}^{\prime}}^{T^{\prime}}=\lambda_{S_{h}^{\prime}}^{T}$. Since $w_{d}(i)-\sum_{h \geq l, i \in S_{h}} \lambda_{S_{h}}^{T^{\prime}} \leq 0$, we have $\forall h \in\{k, \ldots, l-1\}, \lambda_{S_{h}}^{T}=\lambda_{S_{h}^{\prime}}^{T^{\prime}}$ and, therefore, $\forall h \in\{1, \ldots, k-1\}$, $\lambda_{S_{h}^{\prime}}^{T}=\lambda_{S_{h}^{\prime}}^{T^{\prime}}$. Hence $\lambda_{N}^{T}=\lambda_{N}^{T^{\prime}}$.

Consider the bottom case. We suppose that there exists only one section $S_{h}$ containing only item $i$. If there are more than one section of this type, one can easily use the same technique of replacement. Let $N$ be the new created P-node and $N_{1}$ and $N_{2}$ be the two created Q-nodes. Obviously, $\forall h^{\prime} \in\{l, \ldots, m\}, \lambda_{S_{h^{\prime}}^{\prime}}^{T^{\prime}}=\lambda_{S_{h^{\prime}}^{\prime}}^{T}$. By the same arguments as in the upper case of the figure, we have $\forall h^{\prime} \in\{h+1, \ldots, l-1\}, \lambda_{S_{h^{\prime}}^{\prime}}^{T^{\prime}}=\lambda_{S_{h^{\prime}}}^{T}$. Now, since $S_{h-1} \cap S_{h+1}=\emptyset, \forall h^{\prime} \in\{k, \ldots, h-1\}$, $\lambda_{S_{h^{\prime}}^{\prime}}^{T^{\prime}}=\lambda_{S_{h^{\prime}}}^{T}$ and, therefore, $\forall h^{\prime} \in\{1, \ldots, k-1\}, \lambda_{S_{h^{\prime}}^{\prime}}^{T^{\prime}}=\lambda_{S_{h^{\prime}}^{\prime}}^{T}$. In $T^{\prime}, S_{h}^{\prime}=\{i\}$ and the subtree 
rooted in $f_{h}$ is not empty, because otherwise the branch of $T^{\prime}$ containing $S_{h}^{\prime}$ and $f_{h}$ would not represent a maximal clique. Thus $\lambda_{S_{h}^{\prime}}^{T^{\prime}}=\lambda_{f_{h}}^{T}>0$ and $\lambda_{f_{h}}^{T}=\lambda_{f_{h}}^{T^{\prime}}$. We have $\lambda_{N_{1}}=\sum_{h^{\prime}=1}^{h-1} \lambda_{S_{h^{\prime}}}^{T^{\prime}}$ and $\lambda_{N_{2}}=\sum_{h^{\prime}=h+1}^{m} \lambda_{S_{h^{\prime}}}^{T^{\prime}}$. Hence, $\lambda_{N}=\lambda_{N_{1}}+\lambda_{f_{h}}^{T}+\lambda_{N_{2}}=\lambda_{N^{\prime}}^{T^{\prime}}$.

Notice that in both cases of the replacement, every item satisfying the condition of the lemma in $T^{\prime}$, satisfies it also in $T$.

- Suppose $l=m$. Similarly to the case when $l=m$ and $N^{\prime}$ is a P-node, build an MPQ-tree $T$ from $T^{\prime}$. For this purpose, remove $i$ from every section of $N^{\prime}$ containing $i$ and modify $f_{m}$ by inserting $i$ as in the case when $N^{\prime}$ is a P-node. Now, if $S_{m}^{\prime}=\{i\}$, then apply the replacement of Figure 11. The case when $m>3$ (the upper case of the Figure) is straightforward. Consider the case when $m=3$. Recall that by definition of a Q-node, every item of any section must be contained in at least two sections of the same Q-node. Hence, $S_{1}^{\prime} \subset S_{2}^{\prime}, S_{3}^{\prime} \subset S_{2}^{\prime}$ and $S_{2}^{\prime}=S_{1}^{\prime} \cup S_{3}^{\prime}$. Therefore, $S_{1}^{\prime}=S_{2}^{\prime} \backslash\{i\}=A$ and $N^{\prime}$ is replaced by a P-node as shown in the bottom case of the replacement of Figure 11.

Finally, if $f_{m}$ is a leaf we are done. Otherwise, if $i$ does not satisfy the statement of the lemma for $f_{m}$, apply the proof for the new node $f_{m}$.
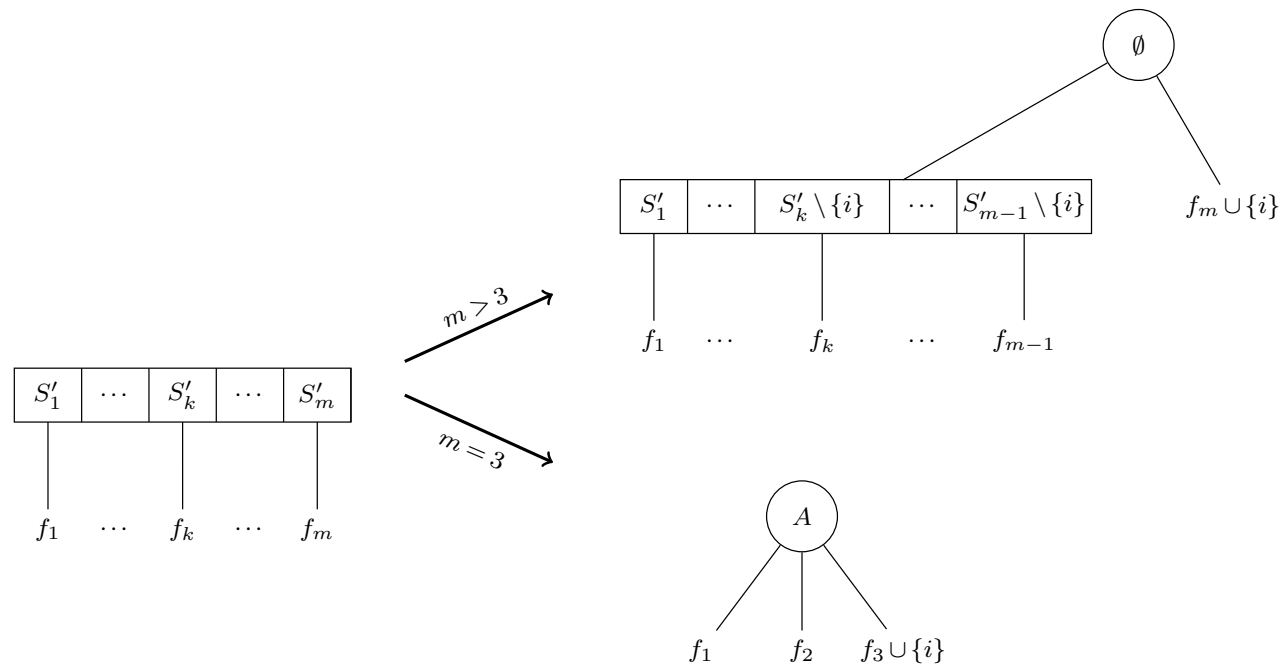

Fig. 11: Q-node replacement when $S_{m}^{\prime}=\{i\}$

By applying the replacement of Figures 9, 10, 11 for all internal nodes (P-type or Q-type) of $T^{\prime}$, for every item $i$ not satisfying the statement of the lemma, we can construct another MPQ-tree associated with a feasible packing in which every item satisfies this statement.

\subsubsection{Order on the children of a node}

Using the general properties of PQ-trees, we impose an order on the children of a node during the generation. We define for any subtree $T_{s}$ of an MPQ-tree $T$ a variable $m_{T}=\min \left\{i \mid i \in T_{s}\right\}$. We say that $T$ is lexicographically ordered if:

- For any two subtrees $T_{1}$ and $T_{2}$ children of the same P-node such that $T_{1}$ is the first in the right to left reading order, we have $m_{T_{1}}>m_{T_{2}}$.

- For two subtrees $T_{1}$ and $T_{2}$ children of the same Q-node where $T_{1}$ is the rightmost and $T_{2}$ is the leftmost, we have $m_{T_{1}}>m_{T_{2}}$.

An example of lexicographically ordered MPQ-trees is depicted in Figure 12. Notice that due to the definition of PQ-trees, for every MPQ-tree, there is an equivalent lexicographically ordered MPQ-tree. Therefore, our algorithm to check feasibility generates all lexicographically ordered possible MPQ-trees. 

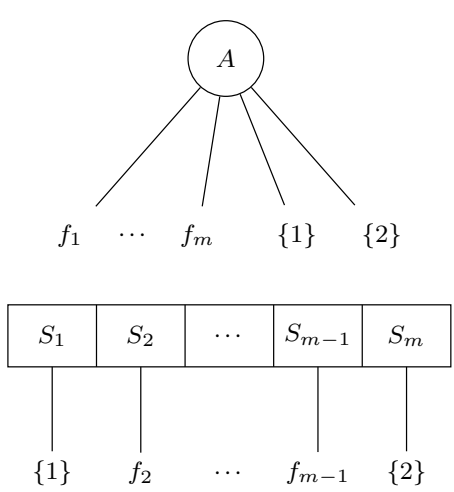
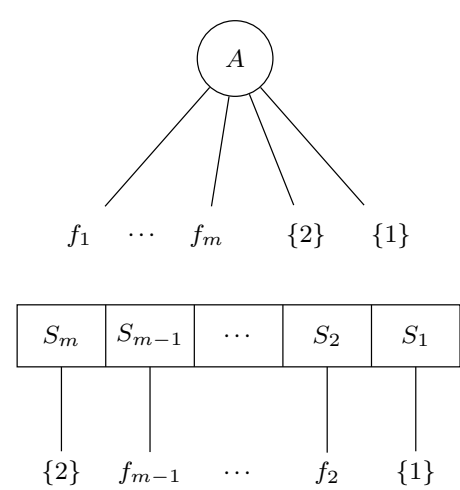

Fig. 12: Lexicographic order between equivalent MPQ-trees

\section{Computational Results}

We report the performance of our algorithm on 37 classical benchmarks for OKP-2 from [3, 2, 7, 12] (Tables 1a) and on 42 benchmarks for OPP-2 defined in [8] (Table 1b). For OKP-2 instances, we used a basic branch-and-bound procedure to select the items to be checked for feasibility.

The program was implemented in Java 6 and was tested on a PC (Pentium IV, 3GHz). These conditions of experimentation are quite similar to the ones used by Fekete and Schepers [12] (PC with Pentium IV processor, $2,8 \mathrm{GHz}$, using $\mathrm{C}++$ ). In contrary to Fekete and Schepers, we do not use some heuristics prior to launching the main algorithm to check feasibility.

Table 1a shows the running times of our algorithm along with the ones existing in the literature, as reported in [12]. The first column (JPV) gives our runtimes. The column FS gives the runtimes of the algorithm from [12]. The column BB corresponds to the algorithm of Baldacci and Boschetti [1], implemented in Visual Digital Fortran 6.0 and run on a Laptop equipped with an Intel Pentium IV, 2.5 GHz. The columns A0, A1, A2 and A3 correspond to the algorithms of Caprara and Monaci, as depicted in [5] and which were implemented in ANSI C and run on a Pentium III $800 \mathrm{MHz}$. We also report the number of unsolved benchmarks (within the time limit of 1800 seconds) and the average time (computed on the set of instances cgcut, gcut and okp), with the convention that an unsolved benchmark counts for 1800 seconds.

The running times of our algorithm are of interest since it is one of the two algorithms to solve all, but one, of the benchmarks within the time limit of 1800 seconds (gcut13 is still open, the optimal value being unknown). Compared to Fekete and Schepers', on an average, our running times turned out to be smaller and were significantly better for 6 instances (cgcut2, gcut3, gcut8, gcut11, gcut12 and okp1), though Fekete and Schepers' algorithm outperforms ours for the 2 instances okp2 and okp5. Additionally, compared to other authors', our running times are considerably better. Note that considering the big difference between our processors and the ones used by Caprara and Monaci for their experiments, one can see that algorithms A1 and A3 are also competitive.

Table $1 \mathrm{~b}$ shows the running times on benchmarks defined in [8]. The third column corresponds to the algorithm of Clautiaux, Carlier and Moukrim [8] implemented in C on a PC (Pentium IV, 2.6 GHz). The size of the containers is $(20,20)$ and there are 10 to 23 items to be packed. These benchmarks are designed to check feasibility (OPP-2) and therefore relevant to the approach described in this paper. However, the size of containers being small, our approach as well as Fekete and Schepers' is less appropriate compared to the methods using a space discretization. The F (resp. N) character in the name of the instance stand for "feasible" (resp. "non feasible") and X character is used when there exists another instance being of the same type (feasible or infeasible) and with the same number of items to be packed. In the case of feasible instances the algorithm has a good performance, giving better execution times than other algorithms for four of them and being significantly worse for only two instances. For unfeasible instances, the running times of about half of them are equivalent to those of the other authors. However, for six instances the running times are less competitive, while only one instance is not solved. In average, our algorithm is thrice faster than Fekete and Schepers', but significantly slower than Clautiaux, Carlier and Moukrim's. 


\begin{tabular}{|c|c|c|c|c|c|c|c|}
\hline Benchmark & $\mathbf{J P V}^{1}$ & $\mathbf{F S}^{2}$ & $\mathrm{BB}^{3}$ & $\mathrm{AO}^{4}$ & $\mathbf{A 1}^{4}$ & $\mathbf{A 2}^{4}$ & $\mathbf{A 3}^{4}$ \\
\hline ngcut1 ... ngcut11 & 0 & 0 & 0 & & & & \\
\hline hccut $2 \ldots$ hccut5 & 0 & 0 & 0 & & & & \\
\hline wang 20 & 0 & 0 & - & 6 & 6 & 17 & 2 \\
\hline cgcut1 & 0 & 0 & 0 & 0 & 1 & 1 & 1 \\
\hline cgcut2 & 96 & $>1800$ & $>1800$ & $>1800$ & $>1800$ & 533 & 531 \\
\hline cgcut3 & 1 & 0 & 95 & 23 & 23 & 4 & 4 \\
\hline gcut1 & 0 & 0 & 0 & 0 & 0 & 0 & 0 \\
\hline gcut2 & 0 & 0 & 0 & 0 & 0 & 25 & 0 \\
\hline gcut3 & $\mathbf{0}$ & 4 & 2 & $>1800$ & 2 & 276 & 3 \\
\hline gcut4 & 137 & 195 & 46 & $>1800$ & 346 & $>1800$ & 376 \\
\hline gcut5 & 0 & 0 & 0 & 0 & 0 & 0 & 0 \\
\hline gcut6 & 0 & 0 & 1 & 0 & 0 & 9 & 0 \\
\hline gcut7 & 0 & 2 & 3 & 1 & 0 & 354 & 1 \\
\hline gcut8 & 60 & 253 & 186 & 1202 & 136 & $>1800$ & 168 \\
\hline gcut9 & 0 & 0 & 0 & 0 & 0 & 0 & 0 \\
\hline gcut10 & 0 & 0 & 0 & 0 & 0 & 6 & 0 \\
\hline gcut11 & 2 & 8 & 3 & 16 & 14 & $>1800$ & 16 \\
\hline gcut12 & 6 & 109 & 12 & 63 & 16 & $>1800$ & 25 \\
\hline gcut13 & $>1800$ & $>1800$ & $>1800$ & $>1800$ & $>1800$ & $>1800$ & $>1800$ \\
\hline okp1 & 1 & 10 & 779 & 24 & 25 & 72 & 35 \\
\hline okp2 & 49 & 20 & 288 & $>1800$ & $>1800$ & 1535 & 1559 \\
\hline okp3 & 1 & 5 & 0 & 21 & 1 & 465 & 10 \\
\hline okp4 & 1 & 2 & 14 & 40 & 2 & 0 & 4 \\
\hline okp5 & 210 & 11 & 190 & 40 & $>1800$ & 513 & 488 \\
\hline \# unsolved & 1 & 2 & 2 & 5 & 4 & 5 & 1 \\
\hline Average time & 64 & 114 & 248 & 474 & 353 & 582 & 228 \\
\hline
\end{tabular}

(a) OKP-2 benchmarks

\begin{tabular}{|l||c|c|c|}
\hline Benchmark & JPV $^{1}$ & FS $^{2}$ & CCM $^{5}$ \\
\hline \hline E02F17 & 30 & 7 & 12 \\
E02F20 & $\mathbf{2}$ & $>1800$ & 12 \\
E02F22 & $\mathbf{2}$ & 167 & 4 \\
E04F15 & 60 & 0 & 1 \\
E04F17 & $\mathbf{9}$ & 13 & 26 \\
E04F19 & 14 & 560 & 7 \\
E04F20 & $\mathbf{0}$ & 22 & 3 \\
E05F15 & $\mathbf{0}$ & 0 & 3 \\
E05F18 & 0 & 0 & 126 \\
E05F20 & 6 & 491 & 2 \\
E07F15 & 0 & 0 & 1 \\
E08F15 & 0 & 0 & 117 \\
E20F15 & 1 & 0 & 1 \\
E00X23 & $>1800$ & $>1800$ & 289 \\
E03X18 & 24 & 0 & 22 \\
E05X15 & 110 & 2 & 0 \\
E07X15 & 79 & 0 & 1 \\
E10X15 & 50 & 0 & 1 \\
E13X15 & 2 & 0 & 0 \\
E20X15 & 8 & 0 & 44 \\
\hline
\end{tabular}

\begin{tabular}{|l||c|c|c|}
\hline E00N10 & 0 & 0 & 0 \\
E00N15 & 2 & 0 & 2 \\
E00N23 & 87 & $>1800$ & 86 \\
E03N10 & 0 & 0 & 1 \\
E03N15 & 0 & 0 & 0 \\
E03N16 & 21 & 0 & 1 \\
E03N17 & 51 & 2 & 32 \\
E04N15 & 45 & 0 & 4 \\
E04N17 & 7 & 0 & 1 \\
E04N18 & 3 & 0 & 1 \\
E05N15 & 7 & 10 & 7 \\
E05N17 & 4 & 0 & 0 \\
E07N10 & 1 & 0 & 1 \\
E07N15 & 0 & 0 & 0 \\
E08N15 & 0 & 0 & 0 \\
E10N10 & 3 & 0 & 1 \\
E10N15 & 0 & 0 & 0 \\
E13N10 & 0 & 0 & 0 \\
E13N15 & 0 & 0 & 0 \\
E15N10 & 0 & 0 & 0 \\
E15N15 & 0 & 0 & 0 \\
\hline \hline \# unsolved & 0 & 0 & 0 \\
\hline Average time & 57 & 3 & 0 \\
\hline
\end{tabular}

(b) OPP-2 benchmarks

Table 1: Running times in seconds

\footnotetext{
1 Java, Pentium IV, 3GHz

${ }^{2} \mathrm{C}++$, Pentium IV, $2.8 \mathrm{GHz}$

3 Visual Digital Fortran 6.0, Pentium IV, $2.5 \mathrm{GHz}$

4 ANSI C, Pentium III, $800 \mathrm{MHz}$

${ }^{5} \mathrm{C}$, Pentium IV, $2.6 \mathrm{GHz}$
} 
Solving the instance gcut13 is still a challenging issue. The particularity of this benchmark is that the size of the container is big (3000 by 3000) while there are many items (32) to be packed, which are of relatively small sizes.

\section{Conclusion}

In this paper we presented a new algorithm for solving the OPP-2 problem by introducing new characterization of feasible packings based on the properties of MPQ-trees. Our approach is of interest since the computational results are competitive and as well as in Fekete and Schepers' case it can be easily extended to the multidimensional case.

\section{References}

1. Baldacci R, Boschetti M (2007) A cutting plane approach for the two-dimensional orthogonal nonguillotine cutting stock problem. European Journal of Operational Research 183(3):1136-1149

2. Beasley J (1985) Algorithms for unconstrained two-dimensional guillotine cutting. Journal of the Operational Research Society 36(4):297-306

3. Beasley J (1985) An exact two-dimensional non-guillotine cutting tree search procedure. Operations Research 33(1):49-64

4. Booth K, Lueker G (1975) Linear algorithms to recognize interval graphs and test for the consecutive ones property. In: Proceedings of the seventh Annual ACM Symposium on Theory of Computing (STOC'75), pp 255-265

5. Caprara A, Monaci M (2004) On the two-dimensional knapsack problem. Operations Research Letters $32(1): 5-14$

6. Carlier J, Clautiaux F, Moukrim A (2007) New reduction procedures and lower bounds for the two-dimensional bin packing problem with fixed orientation. Computers and Operations Research $34(8): 2223-2250$

7. Christofides N, Hadjiconstantinou E (1995) An exact algorithm for orthogonal 2-d cutting problems using guillotine cuts. European Journal of Operational Research 83(1):21-38

8. Clautiaux F, Carlier J, Moukrim A (2007) A new exact method for the orthogonal packing problem. European Journal of Operational Research 183(3):1196-1211

9. Clautiaux F, Jouglet A, Carlier J, Moukrim A (2008) A new constraint programming approach for the orthogonal packing problem. Computers and Operations Research 35(3):944-959

10. Fekete S, Schepers J (1997) On more-dimensional packing i: Modeling. Tech. rep., University of Köln, Germany

11. Fekete S, Schepers J (1997) On more-dimensional packing iii: Exact algorithms. Tech. rep., University of Köln, Germany

12. Fekete SP, Schepers J, van der Veen J (2007) An exact algorithm for higher-dimensional orthogonal packing. Operations Research 55:569-587

13. Ferreira E, Oliveira J (2005) A note on fekete and schepers' algorithm for the non-guillotinable twodimensional packing problem. Technical Report http://paginas.fe.up.pt/ jfo/techreports/ Fekete $\% 20$ and $\% 20$ Schepers $\% 200 \mathrm{PP} \% 20$ degeneracy . pdf

14. Fulkerson D, Gross O (1965) Incidence matrices and interval graphs. Pacific Journal of Mathematics $15(3): 835-855$

15. Ghouila-Houri A (1962) Caractérisation des graphes non orientes dont on peut orienter les aretes de maniere à obtenir le graphe d'une rélation d'ordre. Comptes Rendus Mathématique Académie des Sciences Paris 254:1370-1371

16. Gilmore P, Hoffman A (1964) A characterization of comparability graphs and of interval graphs. Canadian Journal of Mathematics 16:539-548

17. Korte N, Möhring R (1989) An incremental linear-time algorithm for recognizing interval graphs. SIAM Journal on Computing 18(1):68-81

18. Rose D, Tarjan R, Lueker G (1976) Algorithmic aspects of vertex eliminationon graphs. SIAM Journal on Computing 5:266-283 